\title{
ON GROUPS WITH NILPOTENT COMMUTATOR SUBGROUPS
}

\author{
NOBUO INAGAKI
}

\section{§1. Introduction}

It is well known that a supersoluble group has the nilpotent commutator subgroup. But the converse does not hold in general. Therefore, it will be interesting to study the class of groups with nilpotent commutator subgroups. Some results were obtained by B. Huppert [1] and R. Baer [3] on this subject.

It is easily seen that the $p$-length of a group with the nilpotent commutator subgroup is 1 for every $p$ dividing the order of this group. Theorem 1 of this paper may be considered as its local refinement, and is proved in a similar way as B. Huppert [2]. Theorem 2 gives a necessary and sufficient condition for a group to have the nilpotent commutator subgroup in terms of its Hall subgroups. In the last theorem 3 we shall study an interesting class of groups which have maximal subgroups of a certain nature. It turns out that these groups have nilpotent commutator subgroups and admit Sylowtowers.

The auther wishes to express his heartfelt thanks to A. Hattori for his kind leading.

\section{$\S$ 2. Notations and definitions}

$|G| \quad$ order of a finite group $G$.

$G^{\prime} \quad$ commutator subgroup of $G$.

$G^{\prime \prime} \quad$ commutator subgroup of $G^{\prime}$.

$H \subset G \quad H$ is a subgroup of $G$.

$N_{G}(H) \quad$ normalizer of $H$ in $G$.

$C_{G}(H) \quad$ centralizer of $H$ in $G$.

$\emptyset(G) \quad$ Frattini subgroup of $G=$ the intersection of all maximal subgroups of $G$.

$\Delta(G) \quad$ the intersection of all maximal subgroups of $G$ which are not normal in $G$.

Received August 18, 1964. 
Hall subgroup

$p_{j}$-complement $Q_{j}$

$p^{\prime}$-group

$p$-soluble group

$p$-length of $G=l_{p}(G)$

A-group a subgroup whose index is relatively prime to the order of it.

a subgroup $Q_{j}$ such that $|G|=p_{j}^{\alpha}\left|Q_{j}\right|$ and $\left(p_{j},\left|Q_{j}\right|\right)=1$

a group whose order is prime to $p$.

a group, each of whose composition factors is either a $p$-group or a $p^{\prime}$-group.

upper $p$-series of $G$ :

$1=P_{0} \subset N_{0} \subset P_{1} \subset N_{1} \subset P_{2} \subset \cdots \subset P_{i} \subset N_{i} \subset P_{i+1} \subset \cdots$

where $N_{i} / P_{i}$ is the largest normal $p^{\prime}$-subgroup of $G / P_{i}$, and $P_{i+1} / N_{i}$ the largest normal $p$-subgroup of $G / N_{i}$. [8] a soluble group whose Sylow $p$-subgroups are abelian for all $p$ dividing the order of $G$.

\section{$\S 3$. The $p$-length of some $p$-soluble groups}

THEOREM 1. Let $G$ be a p-soluble group. If $P^{\prime}$ is normal in $G$, where $P$ is a Sylow p-subgroup of $G$, then $G$ has p-leugth 1 .

Proof. Our hypothesis holds in all homomorphic images. Let us assume that there exist groups for which the theorem is false for $p$. Then we choose among them a group $G$ of the smallest order.

1) There is one and only one minimal normal subgroup of $G$, and it is a p-group.

For, let $N$ be one of minimal normal subgroups of $G$, then $l_{p}(G / N)=1$, but $l_{p}(G)>1$, so $N$ is a $p$-group. Let $M$ be a minimal normal subgroup which is different from $N$. Then we have $l_{p}(G / M)=1$ and $M \cap N=e$. Now $G / M \cap N=$ $G$ is isomorphic to some subgroup of the direct product $H=G / N \times G / M$. But $l_{p}(H)=1$, since $l_{p}(G / N)=l_{p}(G / M)=1$. It follows $l_{p}(G)=1$ which is a contradiction.

2) There exists a complement $K$ of $N$ in $G$

For, let $M / N$ be the greatest normal subgroup of $G$, whose order is relatively prime to $p$. If $M=N, G / N$ has a normal Sylow $p$-subgroup since $l_{p}(G / N)=1$. $\quad N$ being a $p$-group, we have $l_{p}(G)=1$, which is a contradiction. If $M \supsetneq N$, there exists some subgroup $H$ of $M$ such that $M=N \cdot H$ and $N \cap H=e$, since the order of $N$ is relatively prime to the index of $N$ in $M$. Further, for 
all $g$ in $G, g^{-1} H g$ is conjugate to $H$ in $M$ and we have $G=N_{G}(H) \cdot N$.

If $H$ is a normal subgroup of $G, G$ has that minimal normal subgroup in $H$ which is different from $N$, which contradicts 1$)$. Therefore $N_{G}(H) \subsetneq G$. Let $D=N \cap N_{G}(H)$. Since $N$ is abelian, $D$ is a normal subgroup of $G$. Thus we have $N=D$ or $D=e$. If $D=N$ then $G=N \cdot N_{G}(H)=N_{G}(H)$. This case is excluded from above. Therefore we have $D=e$. Hence $K=N_{\Theta}(H)$ is a complement of $N$ in $G$.

3) $C_{G}(N)=N$.

For, $C_{G}(N)$ splits in the direct product $N \times L$, where $L=C_{G}(N) \cap K . \quad L$ is normal in $K$. since $C_{G}(N)$ is normal in $G$. As $N K=G, L$ is normal in $G$, and we have $L=e$ by 1 ), i.e. $C_{G}(N)=N$.

4) $N$ is not contained in $\Phi(G)$.

For, if $N$ is in $\emptyset(G)$, then $G=N K=\emptyset(G) K=K$. On the other hand $G \equiv K$ since $N \cap K=e$. This is a contradiction.

5) The last part of the proof

Let $P$ be a Sylow $p$-subgroup which contains $N$. From our assumption, we have $P^{\prime} \supset N$ or $P^{\prime}=e$. If $P^{\prime} \supset N$ we have $N \subset P^{\prime} \subset \emptyset(P)$. As $N$ is normal in $G$, we have $N \subset \emptyset(G)$ by Gaschütz [4, Satz 5], which is a contradiction to 4). If $P^{\prime}=e$, i.e. $P$ is abelian, then $C_{G}(N) \supset P \supset N$. Hence $P=N$ by 3$)$. But then we have $l_{p}(G)=1$ which is a contradiction.

Remark. The arguments in 1) 4) are due to Huppert [2, Hilfssatz 1].

Corollary 1. Let $G$ be a soluble group. If each commutator subgroup of all Sylow $p$-subgroups is normal in $G$, then $G$ has p-length 1 for every $p$ dividing $|G|$.

Remark. The symmetric group $S_{1}$ on 4 letters shows that a group $G$ whose factor group $G / N$ by a normal nilpotent subgroup $N$ is an $A$-group has not necessarily $p$-length 1 for every $p$ dividing $|G|$. But $G$ has $p$-length 1 if further $N$ is cyclic or is central in $G$, since we can apply Cor. 1 to these cases.

\section{§4. A necessary and sufficient condition for a group to have the nilpotent commutator subgroup}

Lemma 1. Let $G$ be an A-group. If the commutator subgroups of all Hall subgroups of $G$ are normal in $G$, then $G^{\prime}$ is abelian. 
Proof. Let $|G|=p_{1}^{\alpha_{1}} \cdots p_{n}^{\alpha_{n}}$. Let $P_{j}$ be a $p_{j}$-Sylow subgroup, and $Q_{j}$ be a $p_{j}$-complement, $j=1, \ldots, n$. By our hypothesis $Q_{j}^{\prime}$ is normal in $G$, and we have

$$
\begin{aligned}
& G / Q_{j}^{\prime}=P_{j} Q_{j} / Q_{j}^{\prime} \cong P_{j} Q_{j}^{\prime} / Q_{j}^{\prime} \cdot Q_{j} / Q_{j}^{\prime}, \\
& P_{j} Q_{j}^{\prime} / Q_{j}^{\prime} \cong P_{j}
\end{aligned}
$$

where $P_{j} Q_{j}^{\prime} / Q_{j}^{\prime}, Q_{j} / Q_{j}^{\prime}$ are both abelian groups. Therefore $\left(G / Q_{j}^{\prime}\right)^{\prime}=G^{\prime} / Q_{j}^{\prime}$ is abelian by $N$. Ito [6. Satz 1]. Hence we have

$$
G^{\prime \prime} \subseteq \bigcap_{j} Q_{j}^{\prime} \subseteq \bigcap_{j} Q_{j}=\boldsymbol{e}
$$

TheоReм 2. The commutator subgroup $G^{\prime}$ of $G$ is nilpotent if and only if $G$ is soluole and the commutator subgroups of all Hall subgroups of $G$ are normal in $G$.

Proof. The "only if" part is due to B. Huppert [1. Satz 4]. So we shall prove the "if" part.

1) Our hypothesis holds in $G / P_{i}^{\prime}$.

For, let $H / P_{i}^{\prime}$ be a Hall subgroup of $G / P_{\imath}^{\prime}$.

Case 1. $p_{i}+[G: H]$. $H$ becomes a Hall subgroup in $G$, therefore $H^{\prime}$ is normal in $G$. Then it is clear that $\left(H / P_{i}^{\prime}\right),=H^{\prime} P_{i}^{\prime} / P_{j}^{\prime}$ is normal in $G / P_{i}^{\prime}$.

Case 2. $p_{i} \mid[G: H] . \quad P_{i}^{\prime}$ becomes a normal Hall subgroup in $H$, so there is a Hall subgroup $K$ such that $H=K \cdot P_{i}^{\prime}$ and $K \cap P_{i}^{\prime}=e$. As $K^{\prime}$ is normal in $G,\left(H / P_{i}^{\prime}\right)^{\prime}=P_{i}^{\prime} K^{\prime} / P_{i}^{\prime}$ is normal in $G / P_{i}^{\prime}$.

2) Our hypothesis holds in $G / P_{1}^{\prime} \times \cdots \times P_{n}^{\prime}$.

This results from repeated applications of 1 ).

3) $G^{\prime \prime}$ is nilpotent

For, set $\bar{P}=P_{1}^{\prime} \times \cdots \times P_{n}^{\prime} . \quad G / \bar{P}$ is an $A$-group satisfying our hypothesis. So we can apply Lemma 1 to $G_{i} \bar{P}$, and we obtain $G^{\prime \prime} \subset \bar{P}$, which is nilpotent.

4) The last part of the proof.

Let $G_{p_{i}}^{\prime \prime}$ be the Sylow $p_{i}$-subgroup of $G^{\prime \prime}$. There is a Sylow $p_{i}$-subgroup $P_{i}$ of $G$ which contains $G_{p_{i}}^{\prime \prime}$. The Frattini subgroup $\Phi\left(P_{i}\right)$ contains $P_{i}^{\prime}$. On the other hand, $P_{i}^{\prime} \supset G_{p_{i}}^{\prime \prime}$ by 3), thus $\Phi\left(P_{i}\right) \supset G_{p_{i}}^{\prime \prime}$. Therefore we have $\Phi(G) \supset G_{p_{i}}^{\prime \prime}$ by Gaschütz [4, Satz 5]. This relation holds for all $p_{i}$, so $\Phi(G) \supset G^{\prime \prime}$. It follows that $G^{\prime}$ is nilpotent by B. Huppert (1, Hilfessatz 3 ). 


\section{§5. A certain type of groups having the nilpotent commutator subgroups}

THEOREM 3. Let $S$ be a maximal subgroup of $G$. If $S \cap G^{\prime}$ is in $\Delta(G)$, then i) $[G: S]=a$ power of some prime $p$.

ii) $G^{\prime}$ is nilpotent.

iii) If $P$ is a Sylow p-subgroup of $G$ for $p$ in i), then $P$ is a normal subgroup of $G$.

iv) $G / P$ is nilpotent, and $G$ has a Sylowtower.

Proof. If $S$ is normal in $G$, our assumption means $G^{\prime} \subset \Delta(G)$, or equivalently, $G^{\prime} \subset \emptyset(G)$. Hence our thorem is evident by Wielandt's thorem. Therefore we assume that $S$ is not normal in $G$.

We first prove i) and ii). From the assumption $S \cap G^{\prime} \subset \Delta(G)$, it follows $S \cap \Delta(G) G^{\prime}=\Delta(G)$. Hence if we show that $\Delta(G) G^{\prime} / \Delta(G)$ is a $p$-group, then we have i) $[G: S]=\left[\Delta(G) G^{\prime}: \Delta(G)\right]=$ a power of $p$, and ii) $\Delta(G) G^{\prime}$ is nilpotent by Gaschütz [4, Satz 10]. Therefore we assume that $\Delta(G)=e$, and prove that $G^{\prime}$ is a $p$-group.

Let $a \in S \cap x S x^{-1}$, where $x \notin S$. If $a=x b x^{-1}, b \in S$, we have $a b^{-1} \in S \cap G^{\prime}=e$, i.e. $a=b$. This shows that $x$ commutes with $a$. Since $S \cong G / G^{\prime}$ is abelian, $a$ is then a central element, for $C_{G}(a) \supset\{x, S\}=G$. But, as $\Delta(G)=e$, the only central element is $e$. Hence we have $S \cap x S x^{-1}=e$ for $x \in S$. This means that $G$ is a Frobenius group, and the kernel $G^{\prime}$ is nilpotent by J. Thompson [9, Th. 1]. Since a complement $S$ is a maximal subgroup, $G^{\prime}$ must be characteristic free, and it follows that $G^{\prime}$ is an elementary abelian group of exponet $p$, as desired.

Next we prove iii) and iv). Assume that there is a non-normal maximal subgroup $T$ containing a Sylow $p$-subgroup $P$. Then $\left[\Delta(G) G^{\prime}: \Delta(G) G^{\prime} \cap T\right]$ is a power of $p$, since it divides $\left[\Delta(G) G^{\prime}: \Delta(G)\right]$ which is a power of $p$ by the above. However, [\lrcorner$\left.(G) G^{\prime}: \Delta(G) G^{\prime} \cap T\right]=\left[T G^{\prime}: T\right]$ is relatively prime to $p$, so that this index must be 1 . Hence $T \supset G^{\prime}$, which contradicts the non-normality of $T$. Hence all maximal subgroups containing $P$ are normal in $G$. It follows that iii) $P$ is normal in $G$ by H. Zassenhaus [11, IV. $\$ 1$. Satz 5], and iv) $G / P$ is nilpotent by Wielandt's theorem. It is then clear that $G$ has a Sylowtower.

Remark. T. Ishii and I. Morita have given a following theorem "Let $S$ be 
a maximal subgroup of $G$, if $S \cap G^{\prime}$ is in $\Delta(G)$ and if $[G: S]=p$ then $G$ is supersoluble" [10, Th. 3].

\section{BIBLIOGRAPHY}

[1] B. Huppert, Normalteiler und maximale Untergruppen endlicher Gruppen. Math. Zeit. 60, 409-434 (1954).

[2] B. Huppert, Zur Sylowstruktur auflösbarer Gruppen. Arch. Math. Vol. XII, 161-169 (1961).

[ 3 ] R. Baer, Classes of finite groups and their properties. Illinois J. Vol. 1, 115-187 (1957).

[4] W. Gaschütz, Über die $\Phi$-Untergruppen endlicher Gruppen. Math. Zeit. 58, 160-170 (1953).

[5] A. Hattori, On certain characteristic subgroups of a finite group. Jour. of the Math. Soci. of Japan, Vol. 13, 85-93 (1961).

[6] N. Ito, Über das Produkt von zwei abelschen Gruppen. Math. Zeit. 64, 400-401 (1955).

[7] D. R. Taunt, On A-groups. Proc. Cambridge Phil. Soc. 45, 24-42 (1949).

[8] P. Hall, and G. Higman, On the p-length of $p$-soluble group. Proc. London Math. Soc. 6, 1-42 (1956).

[9] J. Thompson, Finite groups with fixed-point-free automorphisms of prime order. Proc. Nat. Acad. Sci. 45, 578-581 (1959).

[10] T. Ishii, and I. Morita, On the $\Phi_{1}$-group and $\Phi_{2}$-group. Sugaku. Vol. 14, 41-43 (1963) (in Japanese).

[11] H. Zassenhaus, Lehrbuch der Gruppentheorie I. (1937).

Tokyo Electrical Engineering College 